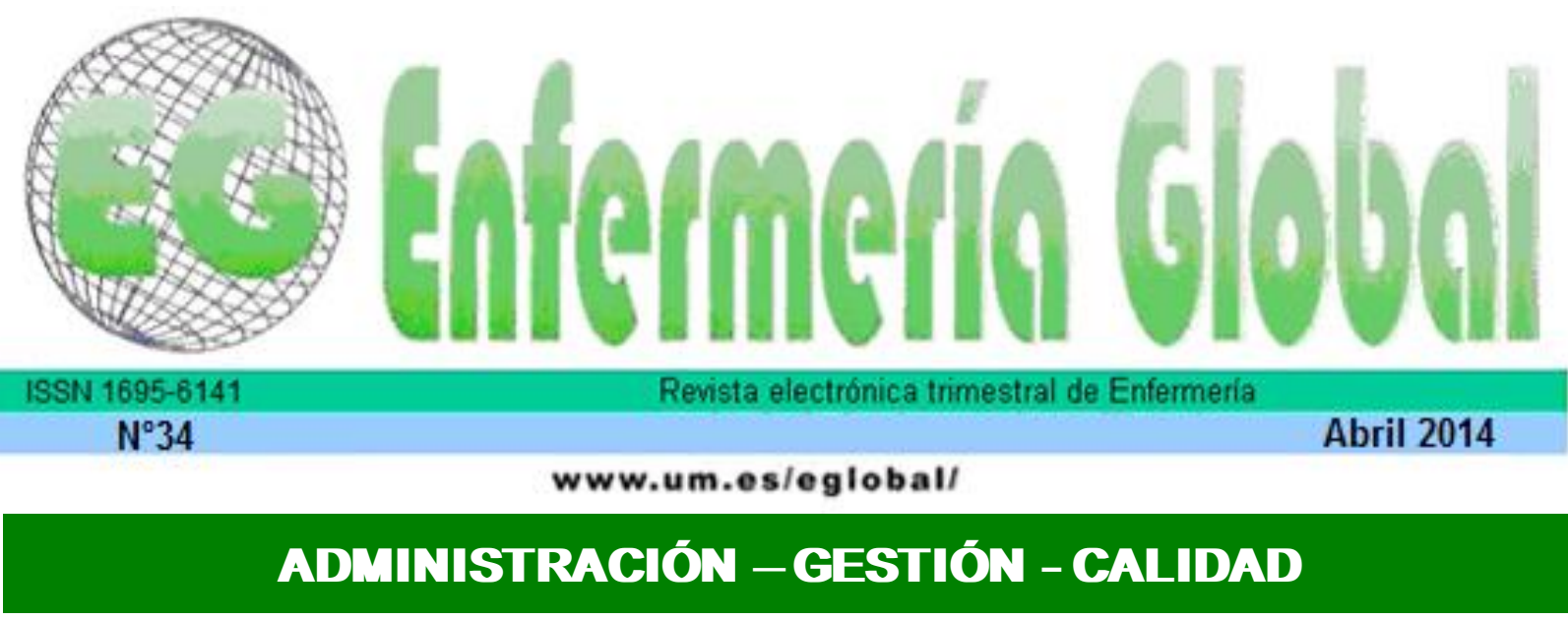

\title{
La atención primaria de salud desde la perspectiva de los usuarios
}

The primary health care from the users perspective

\section{*Giraldo Osorio, Alexandra **Vélez Álvarez, Consuelo}

\begin{abstract}
*Grupo de Investigación Promoción de la Salud y Prevención de la Enfermedad, Programa de Jóvenes Investigadores e Innovadores. E-mail: alegiros@hotmail.com **Departamento de Salud Pública, Grupo de Investigación Promoción de la Salud y Prevención de la Enfermedad. Universidad de Caldas. Colombia.
\end{abstract}

(Artículo producto del Proyecto de Investigación " Estado actual de la estrategia de Atención Primaria de Salud en Manizales" desarrollado en el marco de la Beca de Jóvenes Investigadores e Innovadores de Colciencias (Convocatoria 525), Colombia. Abril 2012 - Abril 2013.)

\begin{abstract}
Palabras clave: Atención primaria de salud; satisfacción del paciente; calidad de la atención de salud; prestación de atención de salud; servicios de salud; atención al paciente.
\end{abstract}

Keywords: Primary health care; patient satisfaction; quality of health care; delivery of health care; health services; patient care.

\section{RESUMEN}

Objetivos: Identificar las características del desarrollo de la estrategia de Atención Primaria de Salud desde la perspectiva de los usuarios y los factores que se relacionan con la utilización de los servicios.

Materiales y métodos: Estudio descriptivo, mixto. Muestra 393 usuarios beneficiarios de la estrategia. La selección de la muestra se realizó según criterios establecidos. Se utilizaron el grupo focal y la encuesta. La información fue categorizada y procesada en el SPSS 15.0.

Resultados: La edad promedio fue 37.62 años. Las principales razones para solicitar una atención fueron recuperarse y prevenir la enfermedad. La edad se asoció significativamente con recuperarse como una razón para solicitar una atención $p<0.05$, el máximo nivel de estudios presentó asociación estadísticamente significativa con recuperarse como una razón para solicitar una atención $p<0.05$ y la percepción del estado de salud se asoció con la satisfacción con la ultima atención que se le ofreció o solicitó $\mathrm{p}<0.05$.

Conclusiones: Las actividades estaban dirigidas a grupos prioritarios. Los medios por los cuales los usuarios se beneficiaron del servicio fueron visita familiar, jornada de salud, unidad móvil y reuniones 
comunitarias. Los factores que se relacionaron con la utilización de los servicios fueron edad, género, accesibilidad geográfica, satisfacción con la última atención recibida, percepción del estado de salud y recuperarse como una razón para solicitar una atención.

\section{ABSTRACT}

Objective: To identify the characteristics of the development of the Primary Health Care strategy according to the users and factors that relate to the use of services.

Materials and methods: Descriptive and mixed study. This study included 393 primary health care users. The sample selection was made according to established criteria. It used the focus group and the survey. The information was categorized and processed in SPSS 15.0.

Results: The average age was 37.62 years. The main reasons for seeking care are recovering and disease prevent. The age was associated significantly with recover as one of the reasons for seeking attention $p<0.05$. The highest level of studies showed statistically significant association with recovery as a reason for requesting attention $p<0.05$ and the health status perception was associated with satisfaction with care last was offered or requested $p<0.05$.

Conclusions: The activities were directed to priority groups. The users benefited from the services through family visit, health day, mobile unit and community meetings. The factors related to services use were age, gender, geographic accessibility, satisfaction with the last care received, health status perception and recovery as one of the reasons for seeking health care.

\section{INTRODUCCIÓN}

La Atención Primaria de Salud (APS) es una estrategia que ha demostrado resultados costo-efectivos sobre las condiciones y calidad de vida de la población. La evaluación de servicios de salud es un tipo de investigación que se dirige a proporcionar información con solidez científica a quienes deben tomar decisiones fundadas en conocimientos válidos en política y planificación sanitaria ${ }^{(1)}$; se constituye como un elemento clave para la mejora continua de la calidad de la atención (2). Algunos autores han hecho énfasis en tomar en cuenta el perfil de los usuarios de la estrategia para orientar los esfuerzos institucionales de cambio ${ }^{(3)}$ y en la necesidad de realizar evaluaciones que integren aspectos relacionados con la diversidad de las funciones que se realizan en el marco de la estrategia de APS. Estudios coinciden en que por medio de la evaluación dirigida a usuarios, ellos otorgan una mayor importancia a aspectos como la disponibilidad de tiempo y servicios, accesibilidad, continuidad de la atención, información que reciben sobre sus problemas de salud o la relación médicopaciente, mientras que ponen menos énfasis en los aspectos administrativos ${ }^{(3,4)}$.

Evaluar la estrategia de APS desde la perspectiva del usuario es básico en todos los ámbitos del sistema de salud ${ }^{(5)}$. Diferentes estudios han evaluado la satisfacción de los usuarios de establecimientos de salud como medida de calidad de la atención, la satisfacción se entiende como la evaluación positiva del paciente sobre la atención médica recibida, esto propicia la adherencia de estos al proveedor de servicios y orienta mejoras en organizaciones de salud ${ }^{(2,3,6,7)}$.

La evaluación de servicios es un ejercicio que promueve la reflexión y el análisis orientado a conducir mejoras en la atención de la salud de la población; con este propósito es útil y relevante realizar evaluaciones a nivel local, nacional e internacional ${ }^{(8-10)}$. 
El objetivo del trabajo es identificar las características del desarrollo de la estrategia de APS valorada desde la perspectiva de los usuarios y los factores que se relacionan con la utilización de los servicios por parte de ellos.

\section{MATERIALES Y MÉTODOS}

Estudio con enfoque mixto que valoró las características de la APS desde la mirada de los usuarios. Como técnicas se utilizaron el grupo de discusión y la encuesta; y como instrumentos, guías de discusión y cuestionarios con preguntas estructuradas y semiestructuradas. La población del estudio estuvo conformada por 72.804 personas beneficiarios de la estrategia ${ }^{(11)}$. El tamaño de la muestra se calculó con un nivel de confiabilidad del 95\%, un margen de error del 5\% y un valor de $p=0,5$ (proporción de personas que son beneficiarias de la estrategia de APS). La muestra final fueron 393 personas. Se realizaron 5 grupos de discusión integrados por mínimo cinco personas y máximo siete, con participación de ambos sexos asistentes a los grupos de los centros de salud, diferentes a los encuestados.

Los criterios de inclusión para la selección de la muestra fueron ser residente del área geográfica para el desarrollo de la estrategia, haber sido beneficiario de la atención en salud y tener 18 o más años de edad. Para la recolección de la información se realizó la prueba piloto en los centros de salud correspondientes a las áreas geográficas priorizadas para el desarrollo de la estrategia y posteriormente se recolectó la totalidad de la información de manera dirigida previa solicitud del consentimiento informado. Para el análisis se categorizó la información cualitativa y retomando las características de la APS. La información cuantitativa fue procesada y analizada en el programa SPSS 15.0. Se realizó análisis descriptivo (tablas de distribución de frecuencias, medidas de dispersión y tendencia central), y análisis bivariado (prueba de $\operatorname{chi}^{2}$ y coeficiente de contingencia, acorde a la naturaleza de la variable).

Con base en la Resolución 08430 de 1993 y la Declaración de Helsinki del 2000, la investigación desarrollada se inscribió dentro de los proyectos sin riesgo. Se contó con la firma del consentimiento informado por parte de los participantes ${ }^{(12,13)}$.

\section{RESULTADOS}

Se incluyeron 393 usuarios que habían recibido atención en el marco de la estrategia de APS en diferentes centros de salud; 389 de sexo femenino y 4 de sexo masculino. La edad mínima fue de 18 años con una máxima de 93 años, el promedio fue de 37.62 años de edad +/-15.7 años. El 51.4\% tenían su lugar de residencia en el estrato socioeconómico 1. El 52.2\% pertenecían a familias de tipo extensa (Tabla I).

Tabla I

Distribución de las variables sociodemográficas. Usuarios de la estrategia de APS

\begin{tabular}{|c|c|c|}
\hline \multicolumn{3}{|c|}{ Género } \\
\hline Femenino & 389 & 99.0 \\
\hline Masculino & 4 & 1.0 \\
\hline Total & 393 & 100.0 \\
\hline \multicolumn{3}{|c|}{ Edad } \\
\hline 15 a 19 años & 55 & 14.0 \\
\hline 20 a 24 años & 52 & 13.2 \\
\hline 25 a 29 años & 53 & 13.5 \\
\hline 30 a 34 años & 36 & 9.2 \\
\hline 35 a 39 años & 35 & 8.9 \\
\hline 40 a 44 años & 37 & 9.4 \\
\hline
\end{tabular}




\begin{tabular}{|c|c|c|}
\hline 45 a 49 años & 28 & 7.1 \\
\hline 50 a 54 años & 33 & 8.4 \\
\hline 55 a 59 años & 17 & 4.3 \\
\hline 60 y más años & 47 & 12.0 \\
\hline Total & 393 & 100.0 \\
\hline \multicolumn{3}{|c|}{ Comuna donde reside } \\
\hline Ciudadela del Norte & 134 & 34.1 \\
\hline La Fuente & 70 & 17.8 \\
\hline San José & 67 & 17.0 \\
\hline Universitaria & 65 & 16.5 \\
\hline La Macarena & 57 & 14.5 \\
\hline Total & 393 & 100.0 \\
\hline \multicolumn{3}{|c|}{ Estrato socioeconómico donde reside } \\
\hline 0 & 5 & 1.3 \\
\hline 1 & 202 & 51.4 \\
\hline 2 & 156 & 39.7 \\
\hline 3 & 30 & 7.6 \\
\hline Total & 393 & 100.0 \\
\hline \multicolumn{3}{|c|}{ Estado civil } \\
\hline Soltera(o) & 145 & 36.9 \\
\hline Casada(o) & 77 & 19.6 \\
\hline Unión libre & 127 & 32.3 \\
\hline Separada(o)/Divorciada(o) & 27 & 6.9 \\
\hline Viuda(o) & 17 & 4.3 \\
\hline Total & 393 & 100.0 \\
\hline \multicolumn{3}{|c|}{ Tipo de familia } \\
\hline Nuclear & 155 & 39.4 \\
\hline Extensa & 205 & 52.2 \\
\hline Monoparental materna & 28 & 701 \\
\hline Ensamblada & 5 & 1.3 \\
\hline Total & 393 & 100.0 \\
\hline \multicolumn{3}{|c|}{ Régimen de afiliación } \\
\hline Subsidiado & 370 & 94.1 \\
\hline Contributivo & 13 & 3.3 \\
\hline Pobre no afiliado & 10 & 2.5 \\
\hline Total & 393 & 100.0 \\
\hline \multicolumn{3}{|c|}{ Máximo nivel de estudios realizados } \\
\hline Sin estudios & 12 & 3.1 \\
\hline Estudios primarios completos & 69 & 17.6 \\
\hline Estudios primarios incompletos & 63 & 16.0 \\
\hline Estudios secundarios completos & 86 & 21.9 \\
\hline Estudios secundarios incompletos & 133 & 33.8 \\
\hline $\begin{array}{l}\text { Estudios técnicos/tecnológicos } \\
\text { completos }\end{array}$ & 16 & 4.1 \\
\hline $\begin{array}{l}\text { Estudios técnicos/tecnológicos } \\
\text { incompletos }\end{array}$ & 5 & 1.3 \\
\hline Estudios universitarios completos & 5 & 1.3 \\
\hline Estudios universitarios incompletos & 4 & 1.0 \\
\hline Total & 393 & 100.0 \\
\hline \multicolumn{3}{|c|}{ Ocupación actual } \\
\hline Estudia & 23 & 5.9 \\
\hline Trabaja dependiente & 55 & 14.0 \\
\hline Trabaja independiente & 35 & 8.9 \\
\hline Realiza actividades domésticas & 280 & 71.2 \\
\hline Total & 393 & 100.0 \\
\hline
\end{tabular}

Fuente: Elaboración propia

Se encontró que las actividades desarrolladas se han llevado a cabo principalmente en grupos prioritarios como niños menores de 10 años de edad, adolescentes, mujeres gestantes y adultos mayores de 65 años. Se resalta que los servicios que les han ofrecido en mayor proporción son educación para adolescentes $(n=265)$ y adultos $(n=251)$, vacunación para mujeres en edad fértil $(n=247)$, servicios amigables/servicio integral al adolescente $(n=213)$, educación para menores de 10 años $(n=202)$, 
atención para salud bucal y educación en higiene oral en adolescentes $(n=173)$, programa de crecimiento y desarrollo $(n=146)$, vacunación para menores de 6 años $(n=144)$, atención para salud bucal y educación en higiene oral en adultos $(n=143)$ y asesorías y consultas en planificación familiar $(n=137)$. Los servicios que han solicitado en mayor proporción han sido atención de las enfermedades más frecuentes en los niños ( $n=201)$, toma de citología vaginal, examen de mama y de testículo en adultos $(n=154)$. En contraste a lo anterior, los servicios que les han ofrecido o que los usuarios han solicitado en menor proporción son atención en salud mental en menores de 10 años y en adultos; atención a personas con discapacidad en menores de 10 años, en adolescentes y en adultos; identificación de casos de maltrato en menores de 10 años, en adolescentes y en adultos; y atención de epidemias en menores de 10 años, en adolescentes y en adultos. Los medios por los cuales los usuarios se habían beneficiado de la atención en salud, fueron visita familiar (100\%), jornada de salud (34.1\%) unidad móvil (27.5\%) y reuniones comunitarias (1.4\%). El $92.4 \%$ informó que el horario en el cual se les realizaba las visitas familiares era adecuado a sus necesidades personales y familiares. En los grupos focales se pudo constatar que el equipo de atención realizaba seguimiento de manera frecuente del estado de salud de los usuarios por medio de las visitas familiares y llamadas telefónicas, ante lo cual los participantes afirmaron que "Por lo general llaman y preguntan si uno se está tomando la droga o a recordar cuando hay una cita pendiente...también cuando los resultados de los exámenes salen malos llaman e informan para explicar que hay que hacer". Las razones por las cuales los usuarios solicitan una atención en salud son principalmente recuperarse y prevenir la enfermedad (47.8\% y $41.2 \%$ respectivamente).

En la Tabla II nótese como entre la edad y recuperarse como una de las razones para solicitar una atención en salud existe asociación estadísticamente significativa y dependencia $\left(\mathrm{Chi}^{2}=32,6 / \mathrm{p}=0,00 /\right.$ Coeficiente de contingencia=0,27). De igual forma al aplicar las pruebas estadísticas, se encontró asociación y dependencia entre el máximo nivel de estudios con recuperarse como una de las razones para solicitar una atención en salud $\left(\mathrm{Chi}^{2}=19,25 / \mathrm{p}=0,001 /\right.$ Coeficiente de contingencia=0,21) (Tabla III). No se encontró asociación y dependencia entre el tipo de familia y las razones para solicitar una atención en salud.

\section{Tabla II}

Edad - Razones para solicitar una atención en salud. Usuarios de la estrategia de APS

\begin{tabular}{|c|c|c|c|c|c|c|c|c|c|}
\hline \multicolumn{10}{|c|}{ Edad x Razones para solicitar una atención en salud } \\
\hline \multirow{2}{*}{ Edad } & \multicolumn{9}{|c|}{ Una de las razones para solicitar una atención en salud es mejorar su salud } \\
\hline & Si & $\%$ & No & $\%$ & Total & $\%$ & $\mathbf{X}^{2}$ & Valor P & Coeficiente \\
\hline $18-29$ & 53 & 33.1 & 107 & 66.9 & 160 & 100.0 & \multirow{4}{*}{2.199} & \multirow{4}{*}{0.53} & \multirow{4}{*}{0.075} \\
\hline $30-44$ & 44 & 40.7 & 64 & 59.3 & 108 & 100.0 & & & \\
\hline $45-64$ & 39 & 39.4 & 60 & 60.6 & 99 & 100.0 & & & \\
\hline $65+$ & 11 & 42.3 & 15 & 57.7 & 26 & 100.0 & & & \\
\hline \multirow{2}{*}{ Edad } & \multicolumn{9}{|c|}{ Una de las razones para solicitar una atención en salud es prevenir la enfermedad } \\
\hline & Si & $\%$ & No & $\%$ & Total & $\%$ & $\mathrm{X}^{2}$ & Valor P & Coeficiente \\
\hline $18-29$ & 73 & 45.6 & 87 & 54.4 & 160 & 100.0 & \multirow{4}{*}{6.323} & \multirow{4}{*}{0.097} & \multirow{4}{*}{0.126} \\
\hline $30-44$ & 34 & 31.5 & 74 & 68.5 & 108 & 100.0 & & & \\
\hline $45-64$ & 45 & 45.5 & 54 & 54.5 & 99 & 100.0 & & & \\
\hline $65+$ & 10 & 38.5 & 16 & 61.5 & 26 & 100.0 & & & \\
\hline \multirow[t]{2}{*}{ Edad } & \multicolumn{9}{|c|}{$\begin{array}{l}\text { Una de las razones para solicitar una atención en salud es fortalecer procesos de } \\
\text { rehabilitación }\end{array}$} \\
\hline & Si & $\%$ & No & $\%$ & Total & $\%$ & $\mathrm{X}^{2}$ & Valor P & Coeficiente \\
\hline $18-29$ & 11 & 6.9 & 149 & 93.1 & 160 & 100.0 & \multirow{4}{*}{2.421} & \multirow{4}{*}{0.49} & \multirow{4}{*}{0.78} \\
\hline $30-44$ & 8 & 7.4 & 100 & 92.6 & 108 & 100.0 & & & \\
\hline $45-64$ & 12 & 12.1 & 87 & 87.9 & 99 & 100.0 & & & \\
\hline $65+$ & 2 & 7.7 & 24 & 92.3 & 26 & 100.0 & & & \\
\hline \multirow{2}{*}{ Edad } & \multicolumn{9}{|c|}{ Una de las razones para solicitar una atención en salud es recuperarse } \\
\hline & Si & $\%$ & No & $\%$ & Total & $\%$ & $\mathbf{X}^{2}$ & Valor P & Coeficiente \\
\hline
\end{tabular}




\begin{tabular}{|c|c|c|c|c|c|c|c|c|c|}
\hline $18-29$ & 53 & 33.1 & 107 & 66.9 & 160 & 100.0 & \multirow{4}{*}{$\begin{array}{c}32.60 \\
1\end{array}$} & \multirow{4}{*}{0.00} & \multirow{4}{*}{0.277} \\
\hline $30-44$ & 52 & 48.1 & 56 & 51.9 & 108 & 100.0 & & & \\
\hline $45-64$ & 63 & 63.6 & 36 & 36.4 & 99 & 100.0 & & & \\
\hline $65+$ & 20 & 76.9 & 6 & 23.1 & 26 & 100.0 & & & \\
\hline
\end{tabular}

Fuente: Elaboración propia

Tabla III

Máximo nivel de estudios - Razones para solicitar una atención en salud. Usuarios de la estrategia de APS

\begin{tabular}{|c|c|c|c|c|c|c|c|c|c|}
\hline \multicolumn{10}{|c|}{ Máximo nivel de estudios - Razones para solicitar una atención en salud es mejorar su salud } \\
\hline \multirow[b]{2}{*}{ Máximo nivel de estudios } & Una & las re & ones & ara sol & itar un & atenció & en sal & es mejo & Ir su salud \\
\hline & Si & $\%$ & No & $\%$ & Total & $\%$ & $x^{2}$ & Valor P & $\begin{array}{l}\text { Coeficient } \\
\text { e }\end{array}$ \\
\hline Sin estudios & 2 & 16.7 & 10 & 83.3 & 12 & 100.0 & \multirow{5}{*}{5.73} & \multirow{5}{*}{0.220} & \multirow{5}{*}{0.120} \\
\hline Estudios primarios & 49 & 37.1 & 83 & 62.9 & 132 & 100.0 & & & \\
\hline Estudios secundarios & 88 & 40.2 & 131 & 59.8 & 219 & 100.0 & & & \\
\hline $\begin{array}{l}\text { Estudios técnicos / } \\
\text { tecnológicos }\end{array}$ & 7 & 33.3 & 14 & 66.7 & 21 & 100.0 & & & \\
\hline Estudios universitarios & 1 & 11.1 & 8 & 88.9 & 9 & 100.0 & & & \\
\hline \multirow{2}{*}{ Máximo nivel de estudios } & \multicolumn{9}{|c|}{$\begin{array}{c}\text { Una de las razones para solicitar una atención en salud es prevenir la } \\
\text { enfermedad }\end{array}$} \\
\hline & Si & $\%$ & No & $\%$ & Total & $\%$ & $x^{2}$ & Valor P & $\begin{array}{l}\text { Coeficient } \\
\text { e }\end{array}$ \\
\hline Sin estudios & 5 & 41.7 & 7 & 58.3 & 12 & 100.0 & \multirow{5}{*}{2.61} & \multirow{5}{*}{0.625} & \multirow{5}{*}{0.081} \\
\hline Estudios primarios & 52 & 39.4 & 80 & 60.6 & 132 & 100.0 & & & \\
\hline Estudios secundarios & 90 & 41.1 & 129 & 58.9 & 219 & 100.0 & & & \\
\hline $\begin{array}{c}\text { Estudios técnicos / } \\
\text { tecnológicos }\end{array}$ & 9 & 42.9 & 12 & 57.1 & 21 & 100.0 & & & \\
\hline Estudios universitarios & 6 & 66.7 & 3 & 33.3 & 9 & 100.0 & & & \\
\hline \multirow{2}{*}{ Máximo nivel de estudios } & \multicolumn{9}{|c|}{$\begin{array}{c}\text { Una de las razones para solicitar una atención en salud es fortalecer } \\
\text { procesos de rehabilitación }\end{array}$} \\
\hline & Si & $\%$ & No & $\%$ & Total & $\%$ & $x^{2}$ & Valor P & $\begin{array}{l}\text { Coeficient } \\
\text { e }\end{array}$ \\
\hline Sin estudios & 0 & 0 & 12 & 100.0 & 12 & 100.0 & \multirow{5}{*}{1.49} & \multirow{5}{*}{0.828} & \multirow{5}{*}{0.061} \\
\hline Estudios primarios & 10 & 7.6 & 122 & 92.4 & 132 & 100.0 & & & \\
\hline Estudios secundarios & 20 & 9.1 & 199 & 90.9 & 219 & 100.0 & & & \\
\hline $\begin{array}{c}\text { Estudios técnicos / } \\
\text { tecnológicos }\end{array}$ & 2 & 9.5 & 19 & 90.5 & 21 & 100.0 & & & \\
\hline Estudios universitarios & 1 & 11.1 & 8 & 88.9 & 9 & 100.0 & & & \\
\hline \multirow[b]{2}{*}{ Máximo nivel de estudios } & \multicolumn{9}{|c|}{ Una de las razones para solicitar una atención en salud es recuperarse } \\
\hline & Si & $\%$ & No & $\%$ & Total & $\%$ & $x^{2}$ & Valor $\mathbf{P}$ & $\begin{array}{l}\text { Coeficient } \\
\text { e }\end{array}$ \\
\hline Sin estudios & 8 & 66.7 & 4 & 33.3 & 12 & 100.0 & \multirow{5}{*}{19.25} & \multirow{5}{*}{0.001} & \multirow{5}{*}{0.216} \\
\hline Estudios primarios & 81 & 61.4 & 51 & 38.6 & 132 & 100.0 & & & \\
\hline Estudios secundarios & 90 & 41.1 & 129 & 58.9 & 219 & 100.0 & & & \\
\hline $\begin{array}{l}\text { Estudios técnicos / } \\
\text { tecnológicos }\end{array}$ & 6 & 28.6 & 15 & 71.4 & 21 & 100.0 & & & \\
\hline Estudios universitarios & 3 & 33.3 & 6 & 66.7 & 9 & 100.0 & & & \\
\hline
\end{tabular}

Fuente: Elaboración propia

El $87.5 \%$ de los usuarios informaron percibían su estado de salud entre bueno y regular. Se encontró asociación y dependencia moderada entre la percepción del estado de salud actual y la satisfacción con la última atención que se le ofreció o que solicitó $\left(\mathrm{Chi}^{2}=30,71 / \mathrm{p}=0,015 /\right.$ Coeficiente de contingencia $\left.=0,269\right)$ (Tabla IV). Los usuarios calificaron como alto y muy alto $(83.7 \%$ el nivel de satisfacción con la última atención en salud que le ofrecieron o que solicito. 


\section{Tabla IV}

Percepción del estado de salud actual - Satisfacción con la ultima atención que se le ofreció o que solicitó. Usuarios de la estrategia de APS

\begin{tabular}{|c|c|c|c|c|c|c|c|c|c|}
\hline \multicolumn{10}{|c|}{ Percepción del estado de salud actual - Satisfacción con la ultima atención que se le ofreció o que solicitó } \\
\hline \multirow{2}{*}{$\begin{array}{c}\text { Percepción del } \\
\text { estado de } \\
\text { salud actual }\end{array}$} & \multicolumn{9}{|c|}{ Satisfacción con la ultima atención que se le ofreció o que solicitó } \\
\hline & $\begin{array}{l}\text { Muy } \\
\text { alto }\end{array}$ & Alto & Medio & Bajo & $\begin{array}{l}\text { Muy } \\
\text { Bajo }\end{array}$ & Total & $x^{2}$ & $\begin{array}{c}\text { Valor de } \\
\mathrm{P}\end{array}$ & Coeficiente \\
\hline Muy bueno & $\begin{array}{c}14 \\
50.0 \% \\
\end{array}$ & $\begin{array}{c}10 \\
35.7 \% \\
\end{array}$ & $\begin{array}{c}0 \\
0 \%\end{array}$ & $\begin{array}{c}1 \\
3.6 \% \\
\end{array}$ & $\begin{array}{c}3 \\
10.7 \% \\
\end{array}$ & $\begin{array}{c}28 \\
100.0 \% \\
\end{array}$ & \multirow{5}{*}{30.71} & \multirow{5}{*}{0.015} & \multirow{5}{*}{0.269} \\
\hline Bueno & $\begin{array}{c}58 \\
25.0 \% \\
\end{array}$ & $\begin{array}{c}144 \\
62.1 \% \\
\end{array}$ & $\begin{array}{c}19 \\
8.2 \% \\
\end{array}$ & $\begin{array}{c}5 \\
2.2 \% \\
\end{array}$ & $\begin{array}{c}6 \\
2.6 \% \\
\end{array}$ & $\begin{array}{c}232 \\
100.0 \%\end{array}$ & & & \\
\hline Regular & $\begin{array}{c}27 \\
24.1 \% \\
\end{array}$ & $\begin{array}{c}61 \\
54.5 \% \\
\end{array}$ & $\begin{array}{c}13 \\
11.6 \% \\
\end{array}$ & $\begin{array}{c}6 \\
5.4 \% \\
\end{array}$ & $\begin{array}{c}5 \\
4.5 \% \\
\end{array}$ & $\begin{array}{c}112 \\
100.0 \% \\
\end{array}$ & & & \\
\hline Malo & $\begin{array}{c}2 \\
12.5 \% \\
\end{array}$ & $\begin{array}{c}9 \\
56.3 \% \\
\end{array}$ & $\begin{array}{c}4 \\
25.0 \% \\
\end{array}$ & $\begin{array}{c}0 \\
0 \% \\
\end{array}$ & $\begin{array}{c}1 \\
6.3 \% \\
\end{array}$ & $\begin{array}{c}16 \\
100.0 \%\end{array}$ & & & \\
\hline Muy malo & $\begin{array}{c}2 \\
40.0 \%\end{array}$ & $\begin{array}{c}2 \\
40.0 \%\end{array}$ & $\begin{array}{c}0 \\
0 \%\end{array}$ & $\begin{array}{c}0 \\
0 \%\end{array}$ & $\begin{array}{c}1 \\
20.0\end{array}$ & $\begin{array}{c}5 \\
100.0 \%\end{array}$ & & & \\
\hline
\end{tabular}

Fuente: Elaboración propia

En cuanto al tiempo que los usuarios tardan en desplazarse desde su lugar de residencia hasta el centro de salud más cercano, el 44,2\% manifestó que es de 0 a 10 minutos, seguido de 11 a 20 minutos en un $38,7 \%$.

En los grupos focales se identificó como fortaleza la labor que realizan las promotoras de salud. Como debilidades la falta de entrega de medicamentos de manera oportuna y la puntualidad (en algunos casos) para la atención de las citas programadas: “...a veces no dan los medicamentos en el centro de salud que a uno le queda más cerquita cuando uno los necesita...". "... uno muchas veces llega 20 minutos antes a la cita que tiene programada, pero lo vienen a atender 30 minutos después, entonces es muy injusto que por cinco minutos que uno se demora de más pierda la cita”.

Se resaltó la falta de continuidad en la atención en salud por barreras para el acceso por parte de las Empresas Promotoras de Salud (EPS): “...No importa si nos dan la remisión aquí, si igual en la EPS no la autorizan...","...las remisiones muchas veces se vencen entonces toca volver a empezar pidiendo cita con el médico general...”.

\section{DISCUSIÓN}

Frente a las características sociodemográficas de los usuarios de la estrategia de APS, el género y la edad condicionan la utilización de los servicios, ya que por una parte pueden actuar como moduladores de la atención ${ }^{(14)}$ y por otro lado pueden ser considerados factores inherentes al estado de salud, particularmente cuando este no se ha controlado perfectamente ${ }^{(15)}$.

Bellón Et al ${ }^{(16)}$ y Mello Et al ${ }^{(17)}$ encontraron que las mujeres utilizan más los servicios de APS que los varones, como se puede constatar en este estudio. Bellón Et al, encontraron que las edades en los que utilizan más estos servicios son entre los 35 y 75 años ${ }^{17}$ en contraste a lo que se encontró en este en el cual las edades de mayor utilización están entre los 18 y los 29 años.

Respecto al tipo de familia al que pertenecen los usuarios de la estrategia, se ha encontrado que el hecho de pertenecer a una familia monoparental se relaciona con una mayor utilización de los servicios de salud ${ }^{(18)}$, diferente a los resultados obtenidos en esta investigación en la cual se observa que con mayor frecuencia la conformación de familias es de tipo extensa y nuclear (52.2\% y $39.4 \%$ respectivamente). 
Otra evidencia científica muestra que existen diferencias en la accesibilidad a los servicios de salud, en especial en el tiempo de espera en la consulta, en función del nivel socioeconómico de los individuos (2). En relación específicamente con la accesibilidad geográfica Acosta $\mathrm{L}$, et al, reportaron que esta fue considerada aceptable por los encuestados, como es el caso de los usuarios participantes en este estudio ${ }^{(2)}$. Investigaciones realizadas han señalado como principal factor condicionante para la utilización de los servicios de APS el nivel educativo de los padres, en especial el de la madre ${ }^{(19)}$ y también han reportado en cuanto a la ocupación, que el hecho de trabajar independiente se relaciona con una menor utilización de los servicios de salud en comparación con el resto de trabajadores ${ }^{(20)}$, en concordancia con los resultados obtenidos en este estudio en el cual se observa una mayor utilización de los servicios por parte de personas que realizan actividades domésticas y que trabajan de manera dependiente $(71.2 \%$ y $14 \%$ respectivamente).

La evidencia muestra que la utilización de los servicios de atención primaria está relacionada principalmente con el estado de salud del individuo, ya sea el autopercibido ${ }^{(17,21,22)}$. En este trabajo se encontró que el $87.5 \%$ de los usuarios calificaron su estado de salud entre bueno y regular.

Respecto a la importancia que los usuarios otorgaron a la promotora de salud por su trabajo con la comunidad, se han encontrado publicaciones en las cuales también se les asigna un nivel de calificación superior sobre otras personas que hacen parte del equipo de salud, dado que son ellas quienes tienen con más frecuencia contacto con las personas, familias y comunidades ${ }^{(23)}$. Estudios han reportado que se han promovido una serie de acciones orientadas a facilitar el acceso a los servicios entre los cuales vale la pena resaltar la gestión para la atención por parte de las EPS ${ }^{(24)}$, similar a lo que el equipo de atención primaria ha llevado a cabo con el fin de brindar continuidad en la atención en salud a los usuarios. Un estudio reportó que el $66.7 \%$ de los pacientes estaba bastante satisfechos o muy satisfechos con la atención recibida ${ }^{(26)}$; similar al nivel de satisfacción con la última atención en salud reportado en este trabajo $(83,7 \%)$.

Frente a la falta de oportunidad para la entrega de los medicamentos, estudios realizados ${ }^{(2,26)}$ han señalado este aspecto como uno de los más críticos. En relación a la continuidad asistencia - accesibilidad entre niveles, existe acuerdo en la literatura que los cambios de nivel asistencial suponen una amenaza para la continuidad de la atención ${ }^{(27,28)}$; esto se pudo evidenciar en los resultados obtenidos sobre la atención de los usuarios en niveles superiores de atención en salud.

\section{CONCLUSIONES}

Entre las principales características de la estrategia de APS se resaltan aspectos como los servicios a los cuales los usuarios han podido acceder en su mayoría están orientados a los niños menores de 10 años de edad, adolescentes, mujeres gestantes, y adultos mayores de 65 años. Los medios por los cuales los usuarios identificaron que han podido acceder a la atención en salud a través de la estrategia de APS han sido principalmente las visitas familiares, las jornadas de salud y la unidad móvil.

Las razones por las que los usuarios han solicitado una atención en salud son principalmente recuperarse y prevenir la enfermedad. Ellos informaron que percibían 
su estado de salud entre bueno y regular. El nivel de satisfacción con la última atención en salud fue de alto y muy alto.

Los factores relacionados con la utilización de los servicios fueron la edad, el género, la accesibilidad geográfica, la satisfacción con la última atención recibida, la percepción del estado de salud y recuperarse como una de las razones para solicitar una atención en salud.

\section{Agradecimientos}

Al programa de Jóvenes Investigadores e Innovadores de Colciencias, al Grupo de Investigación Promoción de la Salud y Prevención de la Enfermedad de la Universidad de Caldas, a la institución de salud en la cual se llevó a cabo el estudio y a los usuarios por su participación.

\section{REFERENCIAS BIBLIOGRÁFICAS}

1. Villalbí JR, Pasarín M, Montaner I, Cabezas C, Starfield B, y Grupo de Trabajo sobre Evaluación en la Atención Primaria de Salud de Barcelona. Evaluación de la atención primaria de salud. Aten Primaria 2003;31:382-385.

2. Acosta L, Burrone MS, Lopez de Neira MJ, Lucchese M, Cometto C, Ciuffolini B, et al. Análisis de la satisfacción del usuario en centros de salud del primer nivel de atención en la provincia de Córdoba, Argentina. Enfermería Global 2011; 21:1-14.

3. Cuba-Fuentes M, Jurado A, Estrella E. Evaluación del cumplimiento de los atributos de la Atención Primaria y grado de satisfacción de los usuarios de un establecimiento de primer nivel de atención. Rev Med Hered 2011; 22(1):4-9.

4. Kerssens JJ, Groenewegen P, Sixma HJ, Boerma WG, Van der Eijk I. Comparison of patient evaluations of health care quality in relation to WHO measures of achievement in 12 European countries. Bull World Health Organ 2004;82:106-14.

5. Briggs C, Capdegelle P, Garner P. Strategies for integrating primary health services in middleand low-income countries: effects on performance, costs and patient outcomes. 1ra ed. The Cochrane Collaboration: Jhon Wiley \& Sons, Ltda; 2006.

6. Pasarín Rua M, Berra S, Rajmil L, Solans M, Borrell C, Starfield B. Un instrumento para la evaluación de la atención primaria de salud desde la perspectiva de la población. Aten Primaria 2007;39(8):395-403.

7. Jiménez Villa J. La evaluación necesita la perspectiva de la población. Aten Primaria 2007; 39(8):395-403.

8. Feldman L, Vivas E, Lugli Z, Alviarez V, Pérez MG, S B. La satisfacción del paciente hospitalario: una propuesta de evaluación. Rev Calid Asist 2007; 22:133-173. 9. Mira JJ, Rodríguez-Marín J, Pesetb R, Ybarra J, Pérez-Jover V, Palazón I, et al. Causas de satisfacción y de insatisfacción de los pacientes en hospitales y atención primaria. Rev Calid Asist 2002; 17:273-283.

10. Redondo Martín S, Bolaños Gallardo E, Almaraz Gómez A, Maderuelo Fernández J. Perceptions and expectations of Primary health care: a new form of identifying improvements. Aten Primaria. 2005; 36:358-366.

11. ASSBASALUD, Secretaría de salud Municipal. Propuesta para el desarrollo de la estrategia Atencion Primaria en Salud en las comunas y corregimientos del Municipio de Manizales, en el marco de las Redes Integradas de Servicios de Salud. Manizales; 2012.

12. Resolución No. 008430 de 4 de octubre de 1993, por la cual se establecen las normas científicas, técnicas y administrativas para la investigación en salud. En: Bogotá D.C: Ministerio de Salud; 1993. 
13. Asociación Médica Mundial. Declaración de Helsinki. Principios éticos para las investigaciones médicas en seres humanos. España: Universidad de Navarra; 2004.

14. Chang W. The meaning and goals of equity in health. J Epidemiol Community Health 2002; 56:488-579.

15. Sáez M. Condicionantes en la utilización de los servicios de atención primaria. Evidencias empíricas e inconsistencias metodológicas. Gac Sanit 2003; 17(5):412417.

16. Bellón J, Delgado A, Luna J, Lardelli P. Influencia de la edad y sexo sobre los distintos tipos de utilización en atención primaria. Gac Sanit 1995;9:343-396.

17. Mello M, Stearns S, Norton E. Do Medicare HMOs still reduce health services use after controlling for selection bias?. Health Econ 2002;11:323-363.

18. Muñoz-Pérez M, Mariscal E, Rubio E, Rey I. Desigualdades sociales en la utilización de servicios de atención primaria: un largo camino por recorrer. Gac Sanit 2000; 14:233-239.

19. Halldórsson M, Kunst A, Köhler L, Mackenbach J. Socioeconomic differences in children's use of physician services in the Nordic countries. J Epidemiol Community Health 2002; 56:200-204.

20. Álvarez B. La demanda atendida de consultas médicas y servicios urgentes en España. Investigaciones Económicas 2001; 25:93-138.

21. Jiménez-Martín S, Labeaga J, Martínez-Granado M. Latent class versus two-part models in the demand for physician services across the European Union. Health Econ 2002; 11:301-322.

22. Vera-Hernández A. Duplicate coverage and demand for health care. The case of Catalonia. Health Econ 1999; 8:579-677.

23. Ruiz-Rodríguez M, Acosta-Ramírez N, Rodríguez Villamizar LA, Uribe LM, LeónFranco M. Experiencia de implementación de un modelo de Atención Primaria. Rev. salud pública $2011 ; 13(6): 885-896$.

24. Mosquera PA, Hernández J, Vega-Romero R, Junca C. Experiencia de implementación de la estrategia de atención primaria en salud en la localidad de Bosa. Rev. Gerenc. Polit. Salud 2011;10(21):124-152.

25. Regidor E, Mateo S, Gutiérrez-Fisach J, Fernández K, Rodríguez C. Diferencias socioeconómicas en la utilización y accesibilidad de los servicios sanitarios en España. Med Clin (Barc) 1996; 107:285-293.

26. Orna Estebana MI, Torrubia Fernández MJ, Peña León I, Isanta Pomar C. Estudio del grado de satisfacción de la asistencia sanitaria recibida en atención primaria, durante los desplazamientos realizados en vacaciones de verano. Aten Primaria 2011; 43(3):158-163.

27. Aller Hernández MB, Vargas Lorenzo I, Sánchez Pérez I, Henao Martínez D, Coderch de Lassaletta J, Llopart López JR, et al. La continuidad asistencial entre niveles percibida por usuarios del Sistema de Salud en Cataluña. Rev Esp Salud Publica 2010; 84:371-387.

28. Gillian P, Anne C, Janet H. Synthesis and conceptual analysis of the SDO Programme's research on continuity of care. York: Report for the National Institute for Health Research service Delivery and Organisation Programme; 2009. SDO Project (08/1813/248).

ISSN 1695-6141

(c) COPYRIGHT Servicio de Publicaciones - Universidad de Murcia 